\title{
A GROUP THEORETIC APPROACH TO GENERALIZED HARMONIC VIBRATIONS IN A ONE DIMENSIONAL LATTICE
}

\author{
J. N. BOYD and P. N. RAYCHOWDHURY \\ Department of Mathematical Sciences \\ Virginia Commonwealth University \\ Richmond, Virginia 23284 U.S.A. \\ (Received July 10, 1984)
}

ABSTRACT. Beginning with a group theoretical simplification of the equations of motion for harmonically coupled point masses moving on a fixed circle, we obtain the natural frequencies of motion for the array. By taking the number of vibrating point masses to be very large, we obtain the natural frequencies of vibration for any arbitrary, but symmetric, harmonic coupling of the masses in a one dimensional lattice. The result is a cosine series for the square of the frequency, $\mathrm{f}_{\mathrm{j}}^{2}=\frac{1}{\pi^{2}} \sum_{\ell=0}^{S} \mathrm{a}(\ell) \cos \ell \beta$ where $0<\beta=\frac{2 \pi j}{\mathrm{~N}} \leq 2 \pi, j \varepsilon\{1,2,3, \ldots, N\}$ and $\mathrm{a}(\ell)$ depends upon the attractive force constant between the $j-$ th and $(j+l)-$ th masses. Lastly, we show that these frequencies will be propagated by wave forms in the lattice.

KEY WORDS AND PHRASES. Harmonic coupling, frequencies of motion, wave forms, lattice. AMS SUBJEC'T CLASSIFICATION CODE. $20 C 35$.

1. INTRODUCTION.

In this paper, we suppose that $\mathrm{N}$ identical, point masses are symmetrically and uniformly arranged around a fixed circle so that the masses are coupled with massless, ideal springs. All motions are confined to the fixed circle. We write potential energy matrices and show how to diagonalize these matrices when the symmetry is that of the rotational group of order $N$. Having begun with quite simple examples, we next take $\mathrm{N}$ to be very large to obtain the natural frequencies for a one dimensional crystal lattice in which both nearest neighboring point masses and next nearest neighbors are coupled by harmonic forces. We then extend this result to include all symmetric couplings and show that the lattice will support wave disturbances of precisely the natural frequencies of the lattice. It should be noted that our results follow from the consideration of the interactions of all pairs of particles around the circular lattice rather than from a Fourier expansion of the potential energy of interaction. This work represents a further use of group theorectic methods which have been reported elsewhere $[1,2,3,4,5]$.

2. POTENTIAL ENERGY MATRICES.

Let us consider the symmetric $N \times N$ matrix $V(N, s)=\left(v_{j k}\right)$ defined in the following manner: $N \geq 3 ; v_{j j}=2$ for $j \varepsilon\{1,2,3, \ldots, N\} ; v_{j k}=-1$ for $k-j \equiv \pm s(\bmod N)$ where 
$s \in\left\{1,2,3, \ldots,\left[\left|\frac{N-1}{2}\right|\right]\right\}$ and $\left[\left|\frac{N-1}{2}\right|\right]$ is the greatest integer less than or equal to $\frac{N-1}{2}$; and $v_{j k}=0$ for all other entries of $v(N, s)$.

For example,

$$
\mathrm{V}(3,1)=\left(\begin{array}{rrr}
2 & -1 & -1 \\
-1 & 2 & -1 \\
-1 & -1 & 2
\end{array}\right), \mathrm{V}(5,1)=\left(\begin{array}{rrrrr}
2 & -1 & 0 & 0 & -1 \\
-1 & 2 & -1 & 0 & 0 \\
0 & -1 & 2 & -1 & 0 \\
0 & 0 & -1 & 2 & -1 \\
-1 & 0 & 0 & -1 & 2
\end{array}\right) \text {, and } \mathrm{V}(6,2)=\left(\begin{array}{rrrrrr}
2 & 0 & -1 & 0 & -1 & 0 \\
0 & 2 & 0 & -1 & 0 & -1 \\
-1 & 0 & 2 & 0 & -1 & 0 \\
0 & -1 & 0 & 2 & 0 & -1 \\
-1 & 0 & -1 & 0 & 2 & 0 \\
0 & -1 & 0 & -1 & 0 & 2
\end{array}\right) \text {. }
$$

The matrix $\mathrm{V}(\mathrm{N}, \mathrm{s})$ arises in writing the elastic potential energies of a symmetric circular array of $\mathrm{N}$ point masses interconnected with ideal springs [1]. If each point mass is coupled to both nearest neighbors but to no other masses, then $s=1$. If each point mass is coupled to its two next nearest neighbors but to no others, then $s=2$.

Consider the case for $\mathrm{N}=4, \mathrm{~s}=1$. Four identical masses move on a fixed frictionless circle. They are connected by springs of force constant $k$ as shown.

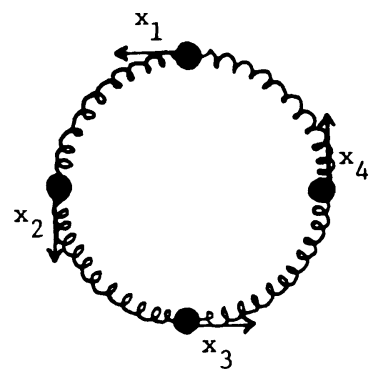

FIGURE 1 .

Let the coordinates, $x_{1}, x_{2}, x_{3}, x_{4}$, denote very small displacements of the masses from their equilibrium positions. All motion is confined to the circle, and the elastic potential energy is P.E. $=\frac{1}{2} k\left[\left(x_{1}-x_{2}\right)^{2}+\left(x_{2}-x_{3}\right)^{2}+\left(x_{3}-x_{4}\right)^{2}+\left(x_{4}-x_{1}\right)^{2}\right]=\frac{1}{2} k \tilde{x} v(4,1) X$ where $X=\left(\begin{array}{l}x_{1} \\ x_{2} \\ x_{3} \\ x_{4}\end{array}\right), \tilde{X}$ is the transpose of $x$, and $v(4,1)$ is as defined.

We can represent the system with a graph on four vertices. The vertices represent the point masses, and two vertices are connected by an edge if and only if the two corresponding masses are coupled by springs.

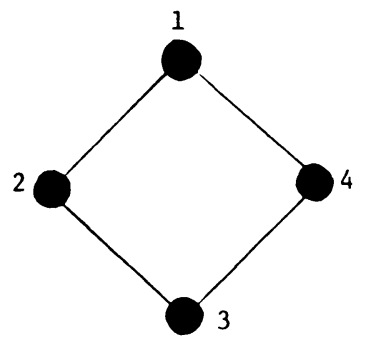

FIGURE 2 . 
Suppose that $N=5, s=2$. That is, each mass is coupled only to its two next nearest neighbors as indicated below. We emphasize that the springs lie along the circle and all motion is on the circle itself.

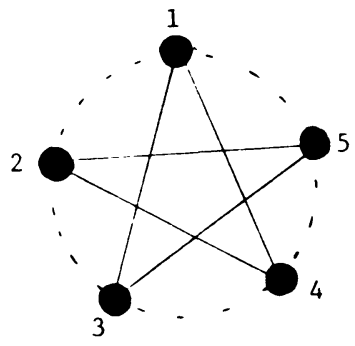

FIGURE 3 .

If the springs have force constant $k$, we have P.E. $=\frac{1}{2} k\left[\left(x_{1}-x_{3}\right)^{2}+\left(x_{2}-x_{4}\right)^{2}+\left(x_{3}-x_{5}\right)^{2}+\left(x_{4}-x_{1}\right)^{2}+\left(x_{5}-x_{2}\right)^{2}\right]=\frac{1}{2} k \tilde{x} V(5,2) x$.

3. GROUP REPRESENTATIONS AND UNITARY TRANSFORMATIONS.

As rotations by $\frac{2 \pi}{N}$ leave the system of $N$ point masses and springs unchanged in so far as kinetic and potential energies are concerned, we take the rotation group $C(N)=\left\{R, R^{2}, \ldots R^{N}\right\}$ where $R$ denotes a rotation of the circle by $\frac{2 \pi}{N}$ to be the symmetry group of the system. As the group is abelian, all irreducible, nonequivalent matrix representations of $\mathrm{C}(\mathrm{N})$ are one dimensional. Thus the $\mathrm{N}$ cyclic groups under complex multiplication which have generators $\exp \left(\frac{2 \pi l i}{N}\right), \ell \varepsilon\{1,2,3, \ldots, N\}$, give all irreducible representations [2].

From these representations we can construct the unitary transformation matrix $U$ which will simultaneously diagonalize $V(N, s)$ for all $s$ :

$$
U=\frac{1}{\sqrt{N}}\left(\begin{array}{cccccccc}
\exp \frac{2 \pi 1}{N} & \exp \frac{4 \pi 1}{N} & \exp \frac{6 \pi 1}{N} & \ldots & \exp \frac{2(N-1) \pi 1}{N} & 1 \\
\exp \frac{4 \pi 1}{N} & \exp \frac{8 \pi 1}{N} & \exp \frac{12 \pi 1}{N} & \ldots & \exp \frac{4(N-1) \pi 1}{N} & 1 \\
\exp \frac{6 \pi 1}{N} & \exp \frac{12 \pi 1}{N} \exp \frac{18 \pi 1}{N} & \ldots & \exp \frac{6(N-1) \pi 1}{N} & 1 \\
\vdots & \vdots & \vdots & & \vdots & \vdots \\
1 & 1 & 1 & \ldots & 1 & 1
\end{array}\right) \text {. }
$$

Since much of what is to follow depends upon this fact, the statement should be justified. Consider the transformed matrix UV(N,s) $U^{-1}$. The j-th row of $U V(N, s)$ is $\frac{1}{\sqrt{N}}\left(\exp \frac{2 \pi j i}{N} \exp \frac{4 \pi j i}{N} \exp \frac{6 \pi j i}{N} \ldots \exp \frac{2 \pi(N-1) j i}{N} \quad 1\right) V(N, s)=\frac{1}{\sqrt{N}}\left(4 \exp \frac{2 \pi j i}{N} \sin ^{2} \frac{\pi s j}{N}\right.$, $\left.4 \exp \frac{4 \pi j i}{N} \sin ^{2} \frac{\pi s j}{N}, 4 \exp \frac{6 \pi j i}{N} \sin ^{2} \frac{\pi s j}{N}, \ldots, 4 \exp \frac{2 N \pi j i}{N} \sin ^{2} \frac{\pi s j}{N}\right)$.

Recalling that $U^{-1}$ is simply the complex conjugate of the transpose of $U$, we can write the $j, k-t h$ entry of $[U V(N, s)] U^{-1}$. This entry is

$$
\left(\frac{4}{N}\right) \sin ^{2} \frac{\pi s j}{N} \sum_{l=1}^{N} \exp \frac{2 \pi(j-k) \ell i}{N}= \begin{cases}4 \sin ^{2} \frac{\pi s j}{N} \text { for } j=k, \\ 0 & \text { for } j \neq k .\end{cases}
$$

The claim has been justified, and $U V(N, s) U^{-1}$ is diagonal for each $s$. 
4. AN APPLICATION.

Let us consider a circular arrangement of $\mathrm{N}$ identical, uniformly spaced particles of mass $\mathrm{m}$. For very large $\mathrm{N}$, we have, in effect, applied the Born condition to transform a linear, one dimensional crystal into a circular array [2]. Suppose that nearest neighbor particles are coupled with ideal, massless springs of force constant $k(1)$ and that next nearest neighbors are coupled with ideal, massless springs of force constant $k(2)$ as indicated below.

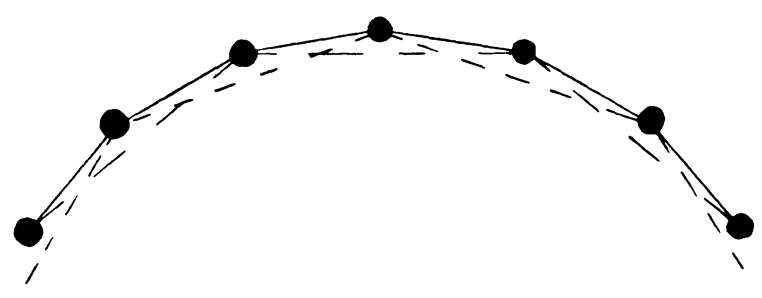

FIGURE 4 .

This configuration would serve as a model for a physical lattice in which harmonic forces between next nearest neighbors were much larger than anharmonic forces between nearest neighbors. In the event that the reader does not wish to visualize such a mechanical arrangement, he could easily interpret the problem in terms of identical LC circuits with capacitors coupled in the appropriate manner.

The Lagrangian for the system is

$$
L=\frac{1}{2} \mathrm{~m}^{2} \mathrm{X} \dot{\mathrm{X}}-\left[\frac{1}{2} \mathrm{k}(1) \tilde{\mathrm{X}} \mathrm{V}(\mathrm{N}, 1) \mathrm{X}+\frac{1}{2} \mathrm{k}(2) \tilde{\mathrm{X}} \mathrm{V}(\mathrm{N}, 2) \mathrm{X}\right]
$$

where $I$ is the $N \times N$ identity matrix, $\dot{\mathrm{x}}=\left(\begin{array}{c}\dot{\mathrm{x}}_{1} \\ \dot{\mathrm{x}}_{2} \\ \vdots \\ \dot{\mathrm{x}}_{\mathrm{N}}\end{array}\right)$, and $\dot{\tilde{x}}=\left(\dot{\mathrm{x}}_{1} \dot{\mathrm{x}}_{2} \ldots \dot{\mathrm{x}}_{\mathrm{N}}\right)$. We can now transform the Lagrangian to diagonalize $\mathrm{V}(\mathrm{N}, 1)$ and $\mathrm{V}(\mathrm{N}, 2): \mathrm{L}=\frac{1}{2} \mathrm{~m} \dot{\tilde{X}} \mathrm{U}^{-1}\left(\mathrm{UIU} \mathrm{U}^{-1}\right) \mathrm{UX}-\left[\frac{1}{2} \mathrm{k}(1) \tilde{\mathrm{X}} \mathrm{U}^{-1}\left(\mathrm{UV}(\mathrm{N}, 1) \mathrm{U}^{-1}\right) \mathrm{UX}\right.$ $\left.+\frac{1}{2} k(2) \tilde{X} U^{-1}\left(U V(N, 2) U^{-1}\right) U X\right]$ $=\frac{1}{2} \dot{m} \cdot I \dot{N}-$

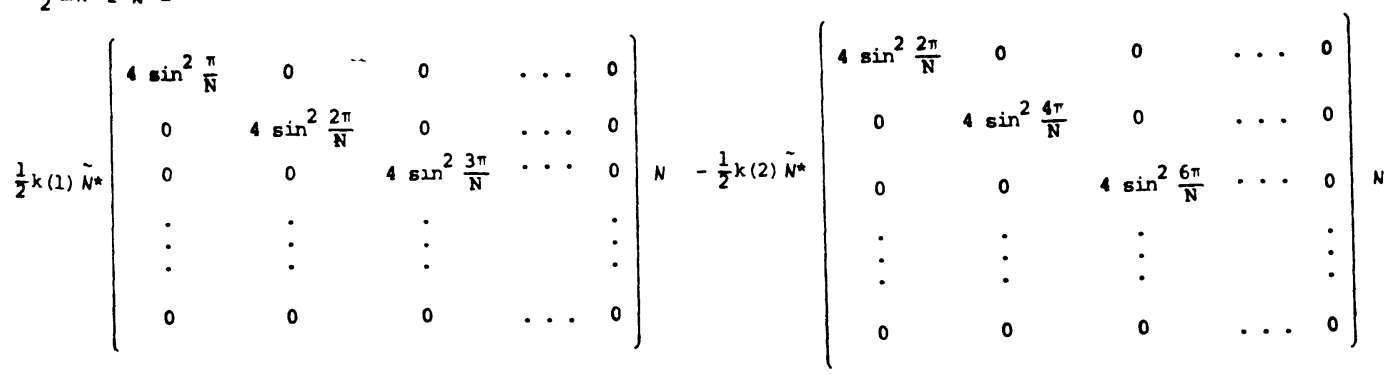

The column matrix $N=$ UX gives the new symmetry coordinates in which all the potential energies are uncoupled. The row matrix $\tilde{N}^{*}$ is the complex conjugate of the transpose of $N$. The equations of motion are given by

$$
\frac{d}{d t}\left(\frac{\partial L}{\partial \dot{n}_{j}^{*}}\right)-\frac{\partial L}{\partial n_{j}^{*}}=0
$$


where $\eta_{j}$ is the $j-t h$ symmetry coordinate and $\eta_{j}^{*}$ is its complex conjugate. Thus we have $m \ddot{n}_{j}+4\left(k(1) \sin ^{2} \frac{j \pi}{N}+k(2) \sin ^{2} \frac{2 j \pi}{N}\right) n_{j}=0$ for $j \in\{1,2,3, \ldots, N\}$.

The natural frequencies are then

$$
f_{j}=\frac{1}{\pi} \sqrt{\frac{k(1) \sin ^{2} \frac{j \pi}{N}+k(2) \sin ^{2} \frac{2 j \pi}{N}}{m}} .
$$

This expression reduces to the well known result for the linear lattice for $k(1)>0$, $k(2)=0[2]$.

5. AN EXTENSION.

It should be clear that if the $N$ point masses are coupled with force constants $k(l) \geq 0$ and potential energy matrices $v(N, l)$ for $\ell \varepsilon\{1,2,3, \ldots, s\}$, then the $j-$ th frequency would become

$$
f_{j}=\frac{1}{\pi} \sqrt{\sum_{\ell=1}^{s}\left[k(\ell) \sin ^{2} \frac{\ell j \pi}{N}\right] / m}
$$

In this event, we can write

$\sum_{\ell=1}^{S} k(\ell) \sin ^{2} \frac{\ell j \pi}{N}=\sum_{\ell=1}^{S} \frac{k(\ell)}{2}\left(1-\cos \ell\left(\frac{2 j \pi}{N}\right)\right) \equiv \sum_{\ell=0}^{s} a(\ell) \cos \ell \beta$ where $a(0)=\sum_{\ell=1}^{s} \frac{k(\ell)}{2}$,

$a(\ell)=-\frac{k(\ell)}{2}$ for $\ell \in\{1,2,3, \ldots, s\}$, and $0<\beta=\frac{2 j \pi}{N} \leq 2 \pi$. The variable $B$ will

be called the wave number of the vibration. If we let $\mathrm{N} \rightarrow \infty$ and $\mathrm{s} \rightarrow \infty$ while assuming absolute convergence for $\sum_{l=1}^{S} \frac{k(l)}{2}$, we have a model in which we can take the harmonic vibrations for all possible symmetric couplings to approximate some frequency distribution obtained from an arbitrary potential energy of interaction.

Supposing that $\mathrm{f}_{j}$ is computed or found experimentally to be $\frac{1}{\pi} \sqrt{\frac{g(\beta)}{m}}$, then we can obtain $a(\ell)$ by noting that $\int_{0}^{2 \pi} g(\beta) \cos \ell \beta d \beta=\int_{0}^{2 \pi} a(\ell) \cos ^{2} \ell \beta d \beta$ and $a(\ell)=\frac{1}{\pi} \int_{0}^{2 \pi} g(B) \cos \ell \beta d B$. Since, the frequency function should be symmetric with respect to $\frac{j \pi}{N}=\frac{\pi}{2}$, our model will be consistent for $g(B)$ symmetric with respect to $B=\pi$, thus explaining the absence of sine terms in the Fourier series.

6. LOCALIZED DISTURBANCES AND WAVE PROPAGATION.

The frequencies obtained are those for the lattice moving as a whole. That is, the frequencies are those of the normal modes of oscillation which involve motions of all constituent parts of the lattice at once.

We now return to the original coordinates in our circular array. These are $\mathbf{x}_{j}$ for $j \in\{1,2,3, \ldots, N\}$ where $x_{j}$ is the displacement of the $j$-th point mass from its equilibrium position. For the moment, let, us take the force constant $k(l)>0$ with $k(s)=0$ if $s \neq l$. Suppose that at $t=0$, the $j-t h$ particle is disturbed. The disturbance will not be registered at the site of the $(j+\ell)$-th particle until some time later. If we postulate that a wave front will move out from the site of the $j$-th particle at $t=0$, then the time required for the front to reach the $(j+l)-t h$ particle will be proportional to $|j+\ell-j|=\ell$. 
Taking the proportionality factor to be $b$, we express this ohysical observation by the introduction of a phase factor $e^{2 \pi b j i / N}$ to multiply the time dependent part of the displacement of the $j-t h$ particle from its equilibrium position. That is, we make the change of variable $x_{j}(t)=e^{2 \pi b j i / N} y_{j}(t)$. Then $x_{j+l}(t) / x_{j}(t)$

$=e^{2 \pi b \ell i / N_{y}}{ }_{j+\ell}(t) / y_{j}(t)$ implies a difference in phase equal to $|b l|$ as required.

From the Lagrangian in the original coordinates, $L=\frac{1}{2} \mathrm{~m} \dot{\mathrm{XIX}}+\frac{1}{2} \mathrm{k}(\ell) \tilde{\mathrm{XV}}(\mathrm{N}, \ell) \mathrm{X}$, we obtain the equation of motion $m \ddot{x}_{j}=k(\ell)\left[-2 x_{j}+x_{j+\ell}+x_{j-\ell}\right]$.

If we now suppose that a periodic wave moves through the array, we write for each $j, x_{j}=e^{2 \tau b j i / N_{y}}(t)=e^{2 \pi b j i / N} Y e^{2 \pi f i t}$ where $Y$ is a constant amplitude. Then the equation of motion becomes $m \ddot{x}{ }_{j}=-m(2 \pi f)^{2} Y e^{2 \pi b j i / N} e^{2 \pi f i t}=k(l) Y e^{2 \pi f i t}\left(e^{2 \pi b(j+l) i / N}\right.$ $\left.-2 e^{2 \pi j i / N}+e^{2 \pi b(j-\ell) i / N}\right)$, or $m(2 \pi f)^{2}=-k(\ell)\left(e^{2 \pi b l i / N}-2+e^{-2 \pi b l i / N}\right)$. Therefore $f^{2}=\frac{k(\ell)}{\pi^{2} m} \sin ^{2}\left(\frac{\pi b l}{N}\right)=\frac{k(l)}{2 \pi^{2} m}\left(1-\cos \left(\frac{2 \pi b}{N} \ell\right)\right)$ or $f=\frac{1}{\pi} \sqrt{\frac{k(l)(1-\cos B l)}{2 m}}$ where $B=\frac{2 \pi b}{N}$.

Thus a longitudinal traveling wave having freuqency of any of the normal modes $(k(l)>0, k(s)=0$ for $\ell \neq s)$ will be supported by the lattice.

Similarly, from the general Lagrangian in the coordinates $x_{j}$, $\mathrm{L}=\frac{1}{2} \mathrm{~m} \dot{\tilde{X}} \mathrm{I} \dot{X}+\frac{1}{2} \sum_{\ell=1}^{S} \mathrm{k}(\ell) \tilde{X} V(N, \ell) X$, we obtain $\mathrm{m}_{j}=\sum_{\ell=1}^{S} k(\ell)\left[-2 x_{j}+x_{j+\ell}+x_{j-\ell}\right]$ implying the existence of a traveling wave form constructed from the superposition of all waves for some fixed $b$ and with the summation taken over $\ell$.

We have $x_{j}=Y e^{2 \pi j i / N} e^{2 \pi f i t}$ where $f^{2}=\frac{1}{\pi^{2} m} \sum_{\ell=1}^{S} k(l) \sin ^{2} \frac{\pi b \ell}{N}=\frac{1}{\pi^{2} m} \sum_{\ell=0}^{S} a(\ell) \cos \ell B$ with $a(\ell)$ and $\beta$ as previously defined, provided that $b$ is an integer.

We conclude with a change to a notation more familiar in solid state physics. Let $u_{0}$ be the equilibrium spacing between successive particles in our array, let $j u_{0}=u$, and let $k=\frac{2 \pi}{N_{0}}$. Then $x_{j}=x(u)=Y e^{k u i} e^{2 \pi f i t}=Y e^{(k u i+2 \pi f i t)} ;$ and, for fixed $b, x(u)$ satisfies the wave equation $\left(\frac{4 \pi^{2} f^{2}}{k^{2}}\right) \frac{\partial^{2} x}{\partial u^{2}}=\frac{\partial^{2} x}{\partial t^{2}}$. Then the velocity of the wave is

$$
\frac{2 \pi \mathrm{f}}{k}=\frac{2}{k} \sqrt{\frac{1}{m} \sum_{\ell=0}^{S} a(\ell) \cos \ell B .}
$$

REFERENCES

1. BOYD, J.N. and RAYCHOWDHURY, P.N., Representation Theory of Finite Abelian Groups Applied to a Linear Diatomic Crystal, International Journal of Mathematics and Mathematical Sciences $\underline{3}$ (1980) 559-74.

2. BOYD, J.N. and RAYCHOWDHURY, P.N., An Application of Projection Operators to a One Dimensional Crystal, Bulletin of the Institute of Mathematics, Academia Sinica 7 (1979) 133-44.

3. BOYD, J.N. and RAYCHOWDHURY, P.N., A One Dimensional Crystal with Nearest Neighbors Coupled Through Their Velocities, ASME Journal of Dynamic Systems Measurement and Control 103 (1981) 293-6.

4. BOYD, J.N. and RAYCHOWDHURY, P.N., Group Representations in Lagrangian Mechanics, Physica 114A(1982) 604-8.

5. BOYD, J.N. and RAYCHOWDHURY, P.N., Two Dimensional Lattice Vibrations from Direct Product Representations of Symmetry Groups, International Journal of Mathematics and Mathematical Sciences, 6(1983) 783-94. 


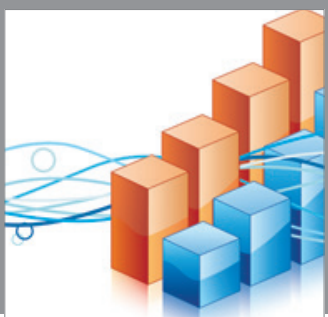

Advances in

Operations Research

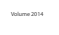

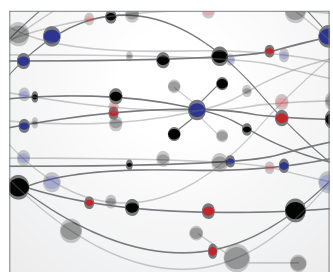

\section{The Scientific} World Journal
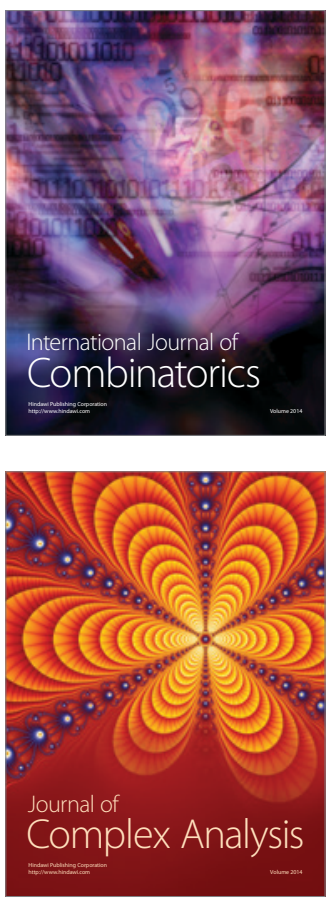

International Journal of

Mathematics and

Mathematical

Sciences
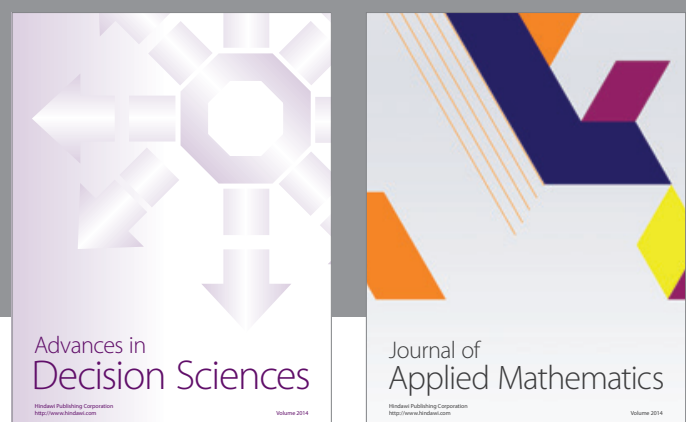

Journal of

Applied Mathematics
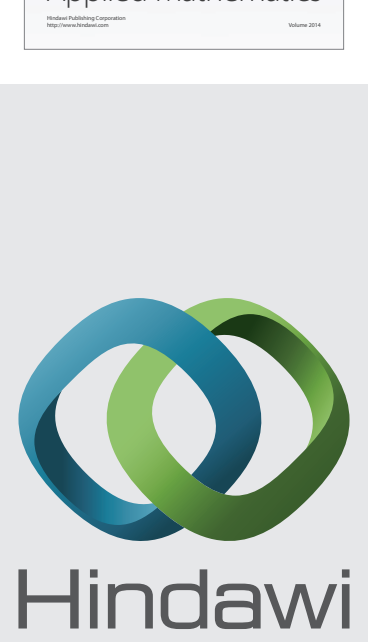

Submit your manuscripts at http://www.hindawi.com
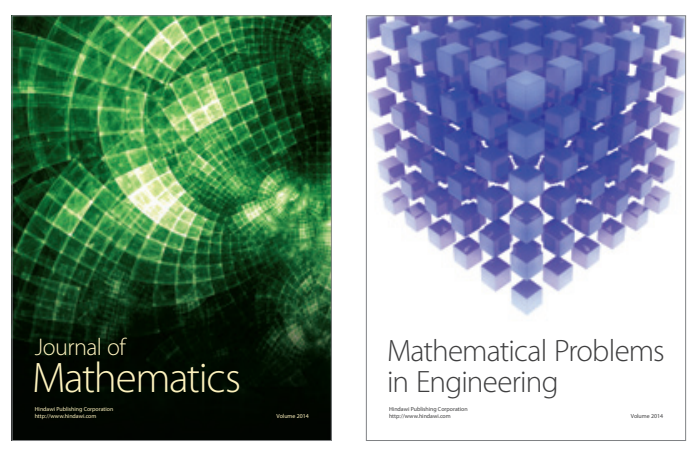

Mathematical Problems in Engineering
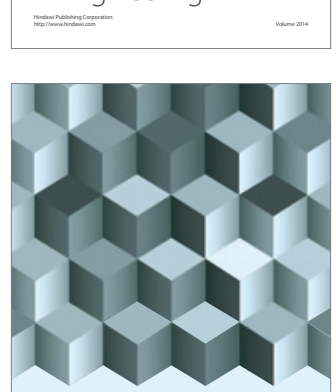

Journal of

Function Spaces
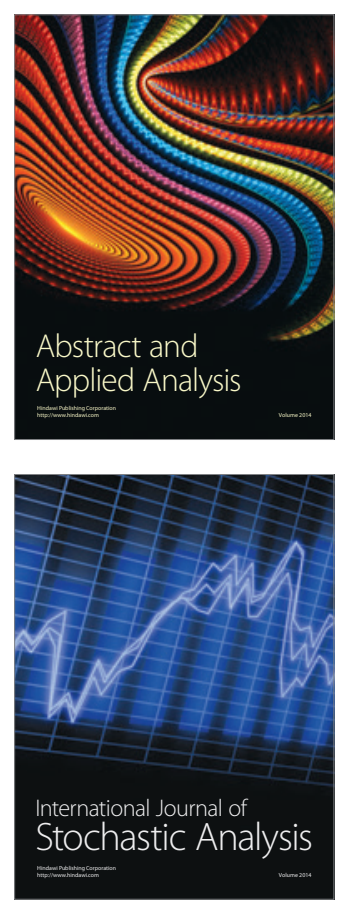

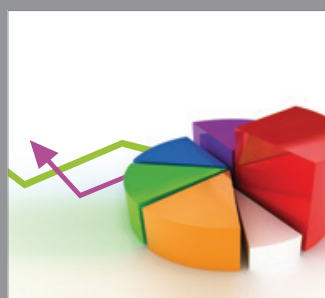

ournal of

Probability and Statistics

Promensencen
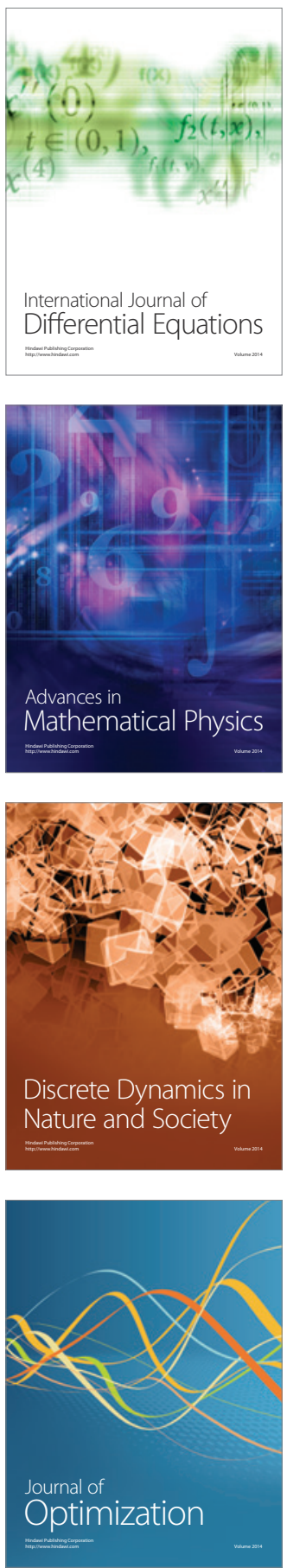